\title{
Investigation Finding
}

National Cancer Institute

\section{Source}

National Cancer Institute. Investigation Finding. NCI Thesaurus. Code C61036.

The result of an examination or inquiry. 